IMAGE

\title{
Insights image for Forskolin attenuates the NLRP3 inflammasome activation and IL-1 $\beta$ secretion in human macrophages
}

Yan Chen ${ }^{1,2}$ and Kari K. Eklund ${ }^{3}$

Pediatric Research (2019) 86:785; https://doi.org/10.1038/s41390-019-0544-z

Nigericin activates the NLRP3 inflammasome by stimulating an intracellular $\mathrm{K}+$ efflux through the pannexin-1 dependent pathway, which induces the assembly of the NLRP3 inflammasome (NLRP3-ASC-Caspase 1) and its subsequent activation of the secretion of IL-1 $\beta$ in PMA-differentiated THP-1 macrophages. Forskolin increases the level of intracellular CAMP, which then binds to NLRP3. Binding of CAMP to NLRP3 promotes the ubiquitination of NLRP3, thus leading into degradation of both CAMP and NLRP3 protein in proteosomes. The degradation of NLRP3 then interrupts the assembly of inflammasome (NLRP3-
ASC-Caspase 1) and thus the activation of pro-caspase 1autocatalysis into caspase 1 which subsequently interrupting the maturation and secretion of IL-1 $\beta$.

\section{REFERENCE}

Chen, $Y$ et al. Forskolin attenuates the NLRP3 inflammasome activation and IL-1 $\beta$ secretion in human macrophages. Pediatr Res. (2019). https://doi.org/10.1038/s41390019-0418-4 [Epub ahead of print].

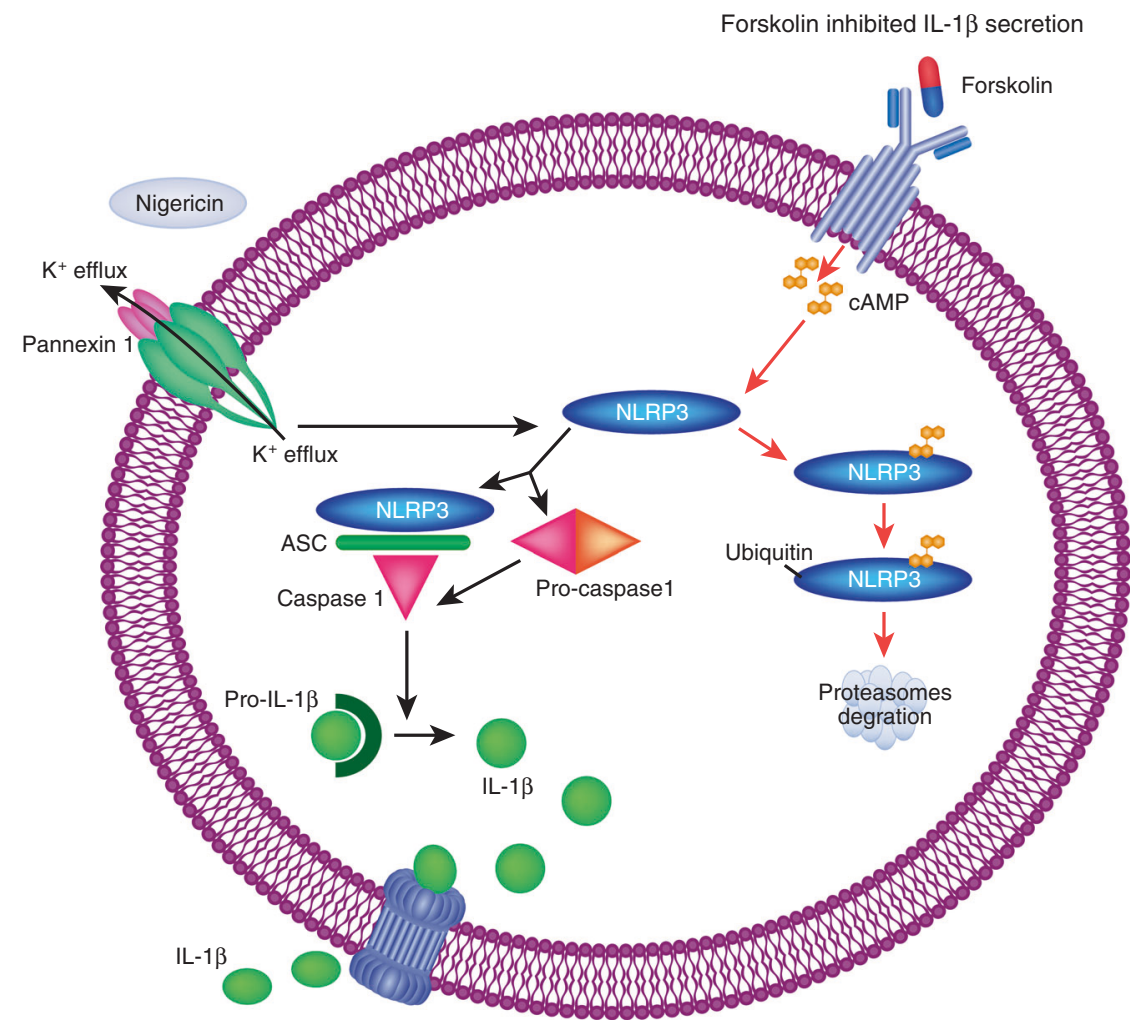

${ }^{1}$ Pediatric urodynamic Center and Department of Urology, Institute of Clinical Medicine, the First Affiliated Hospital of Zhengzhou University, 450052 Zhengzhou, China; ${ }^{2}$ Helsinki Rheumatic Diseases and Inflammation Research Group, Institute of Clinicum, University of Helsinki, Haartmaninkatu 8, 00290 Helsinki, Finland and ${ }^{3}$ Department of Medicine, Division of Rheumatology, Helsinki University Central Hospital, Haartmaninkatu 2, 000290 Helsinki, Finland Correspondence: Yan Chen (chenyan.abby@163.com)

Received: 13 August 2019 Accepted: 13 August 2019

Published online: 21 August 2019 